\title{
Performance Analysis of Both Shot- and Thermal-Noise Limited MultiPulse PPM Receivers in Gamma-Gamma Atmospheric Channels
}

\author{
Ahmed E. Morra, Haitham S. Khallaf, Hossam M. H. Shalaby, Senior Member, IEEE, \\ and Zen Kawasaki, Senior Member, IEEE
}

\begin{abstract}
The performance of free-space optical (FSO) communication systems adopting multipulse PPM (MPPM) techniques is investigated taking into account the effects of both the atmospheric turbulence and receiver noise. The atmospheric turbulence is modeled by a gamma-gamma distribution, which is suitable for both weak and strong turbulence. As for the receiver noise, both shotand thermal-noise limited scenarios are considered. For the shotnoise limited system, both exact and approximate expressions of the average symbol-error rate (SER) of the system are obtained. For the thermal-noise limited system, a closed form for the upper bound of the average system SER, based on the Meijer G function, is obtained. Then, we validate it using Monte Carlo simulation results. Furthermore, we study the effects of changing the atmospheric conditions, operational wavelengths, and number of time slots on the average system performance. In addition, we compare the performance of the aforementioned system with that of the traditional PPM technique, in a gamma-gamma channel, under same constraints on the average energy per bit, transmission data rate, and bandwidth.
\end{abstract}

Index Terms-Atmospheric turbulence, free-space optics, gamma-gamma channels, multipulse pulse-position modulation (MPPM), pulse-position modulation (PPM), shot-noise limited receivers, thermal-noise limited receivers.

\section{INTRODUCTION}

$\mathbf{T}$ HE importance of free-space optical communications (FSO) has increased recently as a result of using it in many telecommunications applications, e.g., satellite links, robotics, last mile connectivity, and optical-fiber backup. Also, it has advantages over radio frequency (RF) communications, e.g., extremely high bandwidth, no spectrum licensing, and interference immunity [1]

Manuscript received April 9, 2013; revised July 30, 2013; accepted August 9, 2013. Date of publication August 14, 2013; date of current version September 13, 2013.

A. E. Morra and H. S. Khallaf are with the Department of Electronics and Communications Engineering, Egypt-Japan University of Science and Technology (E-JUST), Alexandria 21934, Egypt (e-mail: ahmed.morra@ejust.edu eg, ahmedmorra@yahoo.com; haitham.khallaf@ejust.edu.eg, eng.h.khallaf@ gmail.com).

H. M. H. Shalaby is with the Department of Electronics and Communications Engineering, Egypt-Japan University of Science and Technology (E-JUST) Alexandria 21934, Egypt, on leave from the Electrical Engineering Department, Alexandria University, Alexandria 21544, Egypt (e-mail: shalaby@ieee.org).

Z. Kawasaki is with the Graduate School of Engineering, Osaka University, Osaka 565-0871, Japan (e-mail: zen@ @omm.eng.osaka-u.ac.jp).

Color versions of one or more of the figures in this paper are available online at http://ieeexplore.ieee.org.

Digital Object Identifier 10.1109/JLT.2013.2278692
One important phenomenon that degrades the performance of FSO is atmospheric turbulence or scintillation, particularly over ranges greater than $1 \mathrm{~km}$. Scintillation results from inhomogeneities in the temperature and pressure of the atmosphere which would lead to variations in the refractive index. The variations in the refractive index lead to random fluctuations in both the amplitude and phase of the received signal which degrade the performance and lead to an increase in the bit error rate (BER). FSO performance can also be degraded by aerosol scattering effects caused by fog, rain, and snow [2].

The average BER or symbol-error rate (SER) and the outage probability are two metrics that can be used to measure the FSO system performance in fading channels. The outage probability is defined as $P_{\text {out }}=P\left(\right.$ BER $\left.>\mathrm{BER}^{*}\right) \equiv P\left(\mathrm{SNR}<\mathrm{SNR}^{*}\right)$ where $\mathrm{BER}^{*}$ is a predetermined threshold and $\mathrm{SNR}^{*}$ is the average signal to noise ratio in the absence of atmospheric turbulence for a given noise level [3]. In this paper, we use the average SER and BER to measure the FSO system performance in fading channels.

Both ON-OFF keying (OOK) and pulse-position modulation (PPM) formats are popularly used in FSO [4], [5]. The advantage of OOK over PPM is that it is more bandwidth-efficient. However, OOK has many disadvantages, e.g., long sequences of ones or zeros will lead to more synchronization complexity and a decision threshold should be selected appropriately at the receiver side [4], [6]. The energy efficiency of PPM can be increased by increasing the constellation (or frame) size of PPM at the expense of decreasing its bandwidth efficiency. For the aforementioned reasons, multipulse pulse-position modulation (MPPM) has been recently proposed as an alternative modulation technique to PPM and OOK [7]. In fact, MPPM can be considered as a generalization to PPM, where $w \in\{1,2, \ldots, M / 2\}$ pulses are transmitted during a symbol frame of $M$ slots and as a result MPPM is more bandwidth efficient than PPM. In MPPM, bit-tosymbol mapping is carried out as follows. The number of symbol patterns that can be formed for given $M$ and $w$ in MPPM is

$$
H=\left(\begin{array}{l}
M \\
w
\end{array}\right) .
$$

Out of this $H$ symbols, $2^{N}$ symbols are used for mapping and the rest is ignored [8]. Here, $N$ is given by

$$
N=\left\lfloor\log _{2} H\right\rfloor
$$


Furthermore, the relation between MPPM BER and SER is given by [8]:

$$
\mathrm{BER} \leq \frac{1}{2} \frac{2^{N}}{2^{N}-1} \mathrm{SER}
$$

Studies that have investigated the performance of MPPM in free-space optical gamma-gamma channels are very rare. Hamkins and Moision have obtained an exact expression for the SER of MPPM schemes for discrete memoryless channels in nonturbulent atmosphere [9]. Nguyen and Lampe have studied coded MPPM FSO transmission using discrete-time Poisson channel model in nonturbulent atmosphere [4]. They have considered two issues, when MPPM would be better than PPM and how MPPM could be used with error control coding. Gappmair and Muhammad have got an exact expression for the SER of PPM under gamma-gamma optical scintillation model [10]. Xu et al. have discussed the use of binary convolutional coding with iterative detection for the case of MPPM [11], [12]. Wilson et al. have studied the use of multiple-input/multiple-output (MIMO) channel for FSO adopting MPPM [13]. They have concluded that the resulting MIMO channel can reduce FSO turbulent effects in both log-normal and Rayleigh-fading channel models. Balsells et al. have calculated the average BER for a rate adaptive transmission technique using MPPM block coding of variable Hamming weight under turbulence conditions [14]. This type of block coding has a variable amount of pulses and has been shown to have a high peak to average optical power ratio. Their analysis was based on a hyperexponential fitting and Monte Carlo simulation.

Although the MPPM technique is becoming a promising candidate for future free-space optical communications, till now no one has got an exact expression or a closed form expression for the average SER of MPPM in the case of FSO under gamma-gamma distribution. Gamma-gamma distribution has been proposed as a general model that is valid for both weak and strong turbulence [15]. Lognormal and exponential distributions, however, are valid only for weak and strong turbulence, respectively [15], [16].

Our aim in this paper is to derive average SER formulae for FSO systems adopting an intensity modulation/direct detection MPPM technique in gamma-gamma channels for both shot-noise limited and thermal-noise limited cases. In addition, we use that expressions to study the system performance under different design parameters. Specifically, for the shot-noise limited case, we derive both exact and approximate (based on the Gauss-Laguerre quadrature method) expressions for the average SER of this system. We verify our results by noticing that they approach those obtained when using lognormal and exponential channel expressions for both weak and strong turbulence, respectively. Next, for the thermal-noise limited case, we derive a closed form expression for the upper bound of the average SER. Then, we validate it using Monte Carlo simulation results. We use this expression to investigate the effect of changing the wavelength on the performance of the MPPM system under different atmospheric conditions. Also, we study the effect of changing the number of time slots per symbol (modulation level) on the system performance. Finally, we make a compari- son between the average BER performance of both MPPM and PPM for both shot-noise and thermal-noise limited systems.

The rest of the paper is organized as follows. The common statistical channel models, that describe the turbulence effect, are cited in Section II. In Section III, we drive both exact and approximate expressions for the SER of FSO shot-noise limited system adopting MPPM in gamma-gamma channels. We also evaluate the performance of this system numerically and validate the results under different channel models. Section IV is devoted to the derivation of a closed form expression for the upper bound of the SER for the corresponding thermalnoise limited system. We also investigate the effects of different design parameters on this system performance. In Section V, we make a comparison between FSO systems adopting MPPM techniques (in gamma-gamma channels) with those adopting PPM techniques, under the condition of equal data transmission rates. Finally, the conclusion is given in Section VI.

\section{Free-Space Optical Channel Models}

FSO suffers from channel fading, i.e., irradiance fluctuations on the received signal, during the optical signal transmission due to atmospheric turbulence. Several channel models have been assumed to characterize the atmospheric turbulence in the literature. We cite here the most commonly used statistical models.

\section{A. Exponential Channels}

Exponential channel model is suitable for strong atmospheric turbulence. Its probability density function (pdf) is given by [16]

$$
g(I)=\frac{1}{I_{0}} e^{-\frac{I}{I_{0}}}, \quad I \geq 0
$$

where $I$ denotes the intensity of the received optical field and $I_{0}$ denotes its mean, that is, $I_{0}=E\{I\}$, where $E\{\cdot\}$ denotes the expected value.

\section{B. Lognormal Channels}

The lognormal channel model is suitable for weak atmospheric turbulence. Its pdf is given by [16]

$$
g(I)=\frac{1}{I \sigma_{l} \sqrt{2 \pi}} e^{-\frac{\left(\ln I-\mu_{l}\right)^{2}}{2 \sigma_{l}^{2}}}, \quad I \geq 0
$$

where $\mu_{l}$ and $\sigma_{l}^{2}$ denote the mean and variance of $\ln (I)$, respectively. They are related to the mean and variance of $I$ as follows:

$$
\begin{aligned}
\sigma_{l}^{2} & =\ln \left(1+\frac{\operatorname{var}\{I\}}{I_{0}^{2}}\right)=\ln \left(1+\chi_{\mathrm{SC}}\right) \\
\mu_{l} & =\ln \left(I_{0}\right)-\frac{1}{2} \sigma_{l}^{2} .
\end{aligned}
$$

Here, $\chi_{\mathrm{SC}} \stackrel{\text { def }}{=} \operatorname{var}\{I\} / I_{0}^{2}$ denotes the scintillation index and $\operatorname{var}\{\cdot\}$ denotes the variance value.

\section{Gamma-Gamma Channels}

Gamma-Gamma channel model can be considered as a general model and is suitable for both strong and weak atmospheric 
turbulence. Its pdf is given by [15]

$$
\begin{aligned}
g(I)=\frac{2(\alpha \beta)^{\frac{(\alpha+\beta)}{2}}}{I_{0} \Gamma(\alpha) \Gamma(\beta)} & \left(\frac{I}{I_{0}}\right)^{\frac{(\alpha+\beta)}{2}-1} \\
& \quad \times K_{\alpha-\beta}\left(2 \sqrt{\frac{\alpha \beta I}{I_{0}}}\right), \quad I \geq 0
\end{aligned}
$$

where $\Gamma(\cdot)$ is the gamma function, $K_{c}(\cdot)$ is the $c$ th order modified Bessel function of the second kind, and $\alpha$ and $\beta$ are scintillation parameters:

$$
\begin{aligned}
& \alpha=\left(\exp \left[\frac{0.49 \sigma_{R}^{2}}{\left(1+1.11 \sigma_{R}^{\frac{12}{5}}\right)^{\frac{7}{6}}}\right]-1\right)^{-1} \\
& \beta=\left(\exp \left[\frac{0.51 \sigma_{R}^{2}}{\left(1+0.69 \sigma_{R}^{\frac{12}{5}}\right)^{\frac{5}{6}}}\right]-1\right)^{-1} .
\end{aligned}
$$

Here, $\sigma_{R}^{2}=1.23 C_{n}^{2}(2 \pi / \lambda)^{\frac{7}{6}} L^{\frac{11}{6}}$ is unitless Rytov variance, $C_{n}^{2}$ is the refractive-index structure parameter, $L$ is the propagation distance, and $\lambda$ is the operating wavelength. The scintillation index is related to $\alpha$ and $\beta$ as

$$
\chi_{\mathrm{SC}}=\frac{1}{\alpha}+\frac{1}{\beta}+\frac{1}{\alpha \beta} .
$$

As mentioned earlier, in our analysis below, we will be using the gamma-gamma distribution to describe the atmospheric turbulence effect on optical signal transmission.

\section{SHOT-NOISE-LiMITED RECEIVERS}

In this section, we derive both exact and approximate (based on Gauss-Laguerre quadrature method) expressions for the symbol-error rate of shot-noise-limited MPPM systems in gamma-gamma channels. The effect of thermal noise is neglected in this section in order to determine the best we can get from such systems. Shot-noise-limited systems are usually achieved by receivers with high sensitivity, e.g., photomultipliers or photon-counting receivers [17], [18].

\section{A. SER of MPPM Systems in Nonturbulent Atmosphere}

At the receiver side, the detected photon count per MPPM slot follows a Poisson distribution. In this case, the SER is given by [9] with slight modifications:

$$
\begin{aligned}
P_{e \mathrm{Sym}}= & \sum_{K_{\mathrm{min}}=0}^{\infty} \sum_{l=1}^{M-w} \sum_{m=1}^{w}\left(\begin{array}{c}
w \\
m
\end{array}\right)\left(\begin{array}{c}
M-w \\
l
\end{array}\right) p_{1}\left(K_{\mathrm{min}}\right)^{m} \\
& \times\left(1-P_{1}\left(K_{\mathrm{min}}\right)\right)^{w-m}\left[P_{0}\left(K_{\mathrm{min}}\right)^{M-w-l}\right.
\end{aligned}
$$

$$
\begin{aligned}
& \times\left(1-P_{0}\left(K_{\min }\right)\right)^{l}+p_{0}\left(K_{\min }\right)^{l} \\
& \left.\quad \times P_{0}\left(K_{\min }-1\right)^{M-w-l}\left(1-\frac{1}{\left(\begin{array}{c}
l+m \\
m
\end{array}\right)}\right)\right]
\end{aligned}
$$

where $M$ is the number of time slots per symbol, $w$ is the number of signal slots per symbol, and $K_{\text {min }}$ denotes the minimum photon count in symbol signal slots. In addition, $p_{0}(\cdot)$ and $p_{1}(\cdot)$ denote the photon count probabilities of non-signal and signal slots, respectively. Also, $P_{0}(\cdot)$ and $P_{1}(\cdot)$ denote their cumulative distributions. For any $k \in\{0,1,2, \ldots\}$, the last four distributions are given by

$$
\begin{aligned}
& p_{0}(k)=\frac{K_{b}^{k}}{k !} e^{-K_{b}} \\
& p_{1}(k)=\frac{\left(K_{s}+K_{b}\right)^{k}}{k !} e^{-\left(K_{s}+K_{b}\right)} \\
& P_{0}(k)=\sum_{j=0}^{k} \frac{K_{b}^{j}}{j !} e^{-K_{b}} \\
& P_{1}(k)=\sum_{j=0}^{k} \frac{\left(K_{s}+K_{b}\right)^{j}}{j !} e^{-\left(K_{s}+K_{b}\right)}
\end{aligned}
$$

where $K_{s}$ represents the average detected signal photon count per mark slot and $K_{b}$ represents the average detected photon count per slot for background noise.

\section{B. An Exact SER Expression of MPPM Systems in Gamma-Gamma Turbulent Atmosphere}

In our analysis later, we assume that both the transmitter and receiver are fixed and perfectly aligned under a clear atmospheric condition. The effect of scintillation or channel fading is taken into account in our performance derivation. In this case, the average detected signal photons per slot is no longer constant and can be assumed to follow a gamma-gamma distribution:

$$
\begin{aligned}
g\left(K_{s}\right)=\frac{2(\alpha \beta)^{\frac{(\alpha+\beta)}{2}}}{Q \Gamma(\alpha) \Gamma(\beta)}\left(\frac{K_{s}}{Q}\right)^{\frac{(\alpha+\beta)}{2}-1} \\
\quad \times K_{\alpha-\beta}\left(2 \sqrt{\frac{\alpha \beta K_{s}}{Q}}\right), \quad K_{s} \geq 0
\end{aligned}
$$

where $Q=E\left\{K_{s}\right\}$ is the mean of $K_{s}$. The average SER is obtained by averaging $P_{e S y m}$ in (11) with respect to $K_{s}$. Because $K_{s}$ is the only parameter that depends on the channel distribution so $p_{1}\left(K_{\min }\right)^{m}\left(1-P_{1}\left(K_{\min }\right)\right)^{w-m}$ is the only term that will be averaged with respect to $K_{s}$. So the average SER for MPPM under gamma-gamma distribution is obtained by replacing $p_{1}\left(K_{\text {min }}\right)^{m}\left(1-P_{1}\left(K_{\text {min }}\right)\right)^{w-m}$ in (11) by its average $P_{2}\left(K_{\min }\right)$ : 


$$
\begin{aligned}
& P_{2}\left(K_{\min }\right)=\int_{0}^{\infty} p_{1}\left(K_{\min }\right)^{m}\left(1-P_{1}\left(K_{\min }\right)\right)^{w-m} g\left(K_{s}\right) d K_{s} \\
& =\int_{0}^{\infty}\left[\frac{\left(K_{s}+K_{b}\right)^{K_{\min }}}{K_{\min } !} e^{-\left(K_{s}+K_{b}\right)}\right]^{m} \\
& \quad \times\left[1-\sum_{j=0}^{K_{\min }} \frac{\left(K_{s}+K_{b}\right)^{j}}{j !} e^{-\left(K_{s}+K_{b}\right)}\right]^{w-m} g\left(K_{s}\right) d K_{s} .
\end{aligned}
$$

Expanding the brackets and substituting for $g\left(K_{s}\right)$ from (13), we get

$$
\begin{aligned}
P_{2}\left(K_{\min }\right) & \sum_{j_{1}=K_{\mathrm{m} \text { in }}+1}^{\infty} \sum_{j_{2}=K_{\min }+1}^{\infty} \ldots \sum_{j_{w-m}=K_{\min }+1}^{\infty} \\
& \frac{e^{-w K_{b}}}{j_{1} ! j_{2} ! \ldots j_{w-m} ! K_{\min } ! m} \sum_{i=0}^{j_{1}+j_{2}+\ldots+j_{w-m}+m K_{\min }} \\
& \times \frac{2\left(\frac{\alpha \beta}{Q}\right)^{\frac{\alpha+\beta}{2}}}{\Gamma(\alpha) \Gamma(\beta)}\left(j_{1}+j_{2}+\cdots+j_{w-m}+m K_{\min }\right) \\
& \times K_{b}^{j_{1}+j_{2}+\cdots+j_{w-m}+m K_{\mathrm{min}}-i} \int_{0}^{\infty} K_{s}^{i+\frac{\alpha+\beta}{2}-1} \\
& \times e^{-w K_{s}} K_{\alpha-\beta}\left(2 \sqrt{\frac{\alpha \beta K_{s}}{Q}}\right) d K_{s} .
\end{aligned}
$$

The last integration can be evaluated using [19] as follows:

$$
\begin{gathered}
\int_{0}^{\infty} K_{s}^{i+\frac{(\alpha+\beta)}{2}-1} e^{-w K_{s}} K_{\alpha-\beta}\left(2 \sqrt{\frac{\alpha \beta K_{s}}{Q}}\right) d K_{s} \\
=\frac{\Gamma(i+\alpha) \Gamma(i+\beta)}{2 \sqrt{\frac{\alpha \beta}{Q}}} e^{\left(\frac{\alpha \beta}{2 w Q}\right)} w^{-\left(i+\frac{(\alpha+\beta)}{2}-\frac{1}{2}\right)} \\
\times W_{-\left(i+\frac{(\alpha+\beta)}{2}-\frac{1}{2}\right), \frac{1}{2}(\alpha-\beta)}\left(\frac{\alpha \beta}{w Q}\right)
\end{gathered}
$$

where $W_{Q, \mu}(z)$ is the Whittaker function [19]. Substituting back into (15), we get (17) at the bottom of this page, where for any integer $j \geq(w-m)\left(K_{\min }+1\right), r(j)$ is defined as the summation

$$
r(j)=\sum_{\left(s_{1}, s_{2}, \ldots, s_{w-m}\right) \in \mathcal{X}(j)} \frac{1}{s_{1} ! s_{2} ! \ldots s_{w-m} !}
$$

over the set of vectors $\mathcal{X}(j)$ :

$$
\begin{aligned}
\mathcal{X}(j) \stackrel{\text { def }}{=} & \left\{\left(s_{1}, s_{2}, \ldots, s_{w-m}\right) \in \mathbb{N}^{w-m}:\right. \\
& \sum_{i=1}^{w-m} s_{i}=j \text { and } \forall \ell \in\{1,2, \ldots, w-m\} \\
& \left.K_{\min }+1 \leq s_{\ell} \leq j-(w-m-1)\left(K_{\min }+1\right)\right\} .
\end{aligned}
$$

\section{An Approximate SER Expression of MPPM Systems in Gamma-Gamma Turbulent Atmosphere}

The computation complexity of the exact SER expression given in the last Section is high and time consuming. This motivates us to obtain an approximate expression based on the Gauss-Laguerre quadrature rule [20]. This method produces faster results with good approximation. Using this method, it is easy to show that from (14):

$$
\begin{aligned}
& P_{2}\left(K_{\min }\right) \\
& =\frac{e^{-w K_{b}}}{K_{\min } !^{m}} \int_{0}^{\infty} e^{-w K_{s}}\left(K_{s}+K_{b}\right)^{m K_{\min }} \\
& \quad \times\left[e^{\left(K_{s}+K_{b}\right)}-\sum_{j=0}^{K_{\min }} \frac{\left(K_{s}+K_{b}\right)^{j}}{j !}\right]^{w-m} g\left(K_{s}\right) d K_{s} .
\end{aligned}
$$

According to the generalized Gauss-Laguerre quadrature rule:

$$
\int_{a}^{\infty} W(x) f(x) d x \approx \sum_{i=1}^{c} \Lambda_{i} f\left(V_{i}\right)
$$

where $c>1$ denotes the number of terms; and for any $i \in\{1,2, \ldots, c\}, V_{i}$ is the $i$ th root of Laguerre polynomial $L_{c}(x)$ with degree $c$ and $\Lambda_{i}$ is the corresponding weighting coefficient [20]. Here, the weighting function $W(x) \stackrel{\text { def }}{=}$ $(x-a)^{\alpha_{2}} e^{-b(x-a)}$, where $a, b$, and $\alpha_{2}$ are constants. In our

$$
P_{2}\left(K_{\min }\right)=\left\{\begin{array}{cc}
\sum_{i=0}^{m K_{\min }} \frac{\left(\frac{\alpha \beta}{Q}\right)^{\frac{\alpha+\beta-1}{2}}}{\Gamma(\alpha) \Gamma(\beta)}\left(\begin{array}{c}
K_{\min } \\
i
\end{array}\right) e^{\left(-w K_{b}\right)} K_{b}^{m K_{\min }-i} \frac{\Gamma(i+\alpha) \Gamma(i+\beta)}{K_{\min } !^{(m}} e^{\left(\frac{\alpha \beta}{2 w Q}\right)} w^{-\left(i+\frac{(\alpha+\beta)}{2}-\frac{1}{2}\right)} & \text { for } \mathrm{w}=\mathrm{m} \\
\times W_{-\left(i+\frac{(\alpha+\beta)}{2}-\frac{1}{2}\right), \frac{1}{2}(\alpha-\beta)}\left(\frac{\alpha \beta}{w Q}\right) ; & \\
\sum_{j=(w-m)\left(K_{\min }+1\right)}^{\infty} \sum_{i=0}^{j+m K_{\min }} \frac{\left(\frac{\alpha \beta}{Q}\right)^{\frac{\alpha+\beta-1}{2}}}{\Gamma(\alpha) \Gamma(\beta)}\left(\begin{array}{c}
j+m K_{\min } \\
i
\end{array}\right) e^{\left(-w K_{b}\right)} K_{b}^{j+m K_{\min }-i} & \text { for } \mathrm{w} \neq \mathrm{m} .
\end{array}\right.
$$




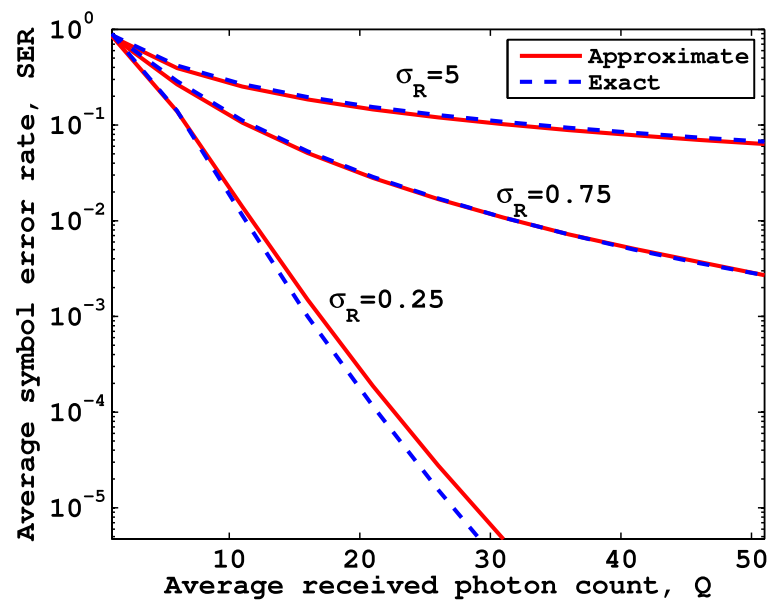

Fig. 1. Average symbol-error rate (SER) versus average received signal photon count $Q$ using both exact and approximate methods under gamma-gamma distribution with $M=8, w=4$, and $K_{b}=1$.

case, we set $a=0, b=w, \alpha_{2}=0$, and $x=K_{s}$. This gives $W\left(K_{s}\right)=e^{-w K_{s}}$, and then, we find that:

$$
\begin{aligned}
P_{2}\left(K_{\min }\right) \approx \sum_{i=1}^{c} & \Lambda_{i} \frac{e^{-w K_{b}}}{K_{\min } !^{m}}\left(V_{i}+K_{b}\right)^{m K_{\min }} \\
\times & {\left[e^{\left(V_{i}+K_{b}\right)}-\sum_{j=0}^{K_{\min }} \frac{\left(V_{i}+K_{b}\right)^{j}}{j !}\right]^{w-m} g\left(V_{i}\right) . }
\end{aligned}
$$

\section{Numerical Results}

In this section, we numerically evaluate both exact and approximate expressions of the symbol-error rates obtained in the previous sections for different channel parameters. In our evaluation, we use MPPM frames of size $M=8$ slots and $w=4$ pulses per frame. The average photon count for background noise is assumed to be $K_{b}=1$.

In Fig. 1, we compare between the exact and approximate expressions under gamma-gamma atmospheric channel with weak, moderate, and strong turbulence conditions of $\sigma_{R}=0.25,0.75$, and 5 , respectively. We notice from the figure that both exact and approximate expressions are nearly identical but the computation complexity is much reduced when using the latter.

Fig. 2 illustrates the change of average SER with the average received photon count $Q$ under gamma-gamma channel. In our evaluations, we have used the approximate method with Laguerre polynomial degree $c=50$. We have studied weak, moderate, and strong turbulence conditions of $\sigma_{R}=0.25,0.75$, and 5, respectively. The symbol-error rates of the MPPM system under both exponential and lognormal channels (which are valid only for strong and weak turbulence, respectively) are also included in the figure for the sake of comparison. The variance of the lognormal distribution is governed by the same scintillation index as that of the gamma-gamma model, which is equal to $0.062,0.475$, and 1.214 for weak, moderate and, strong

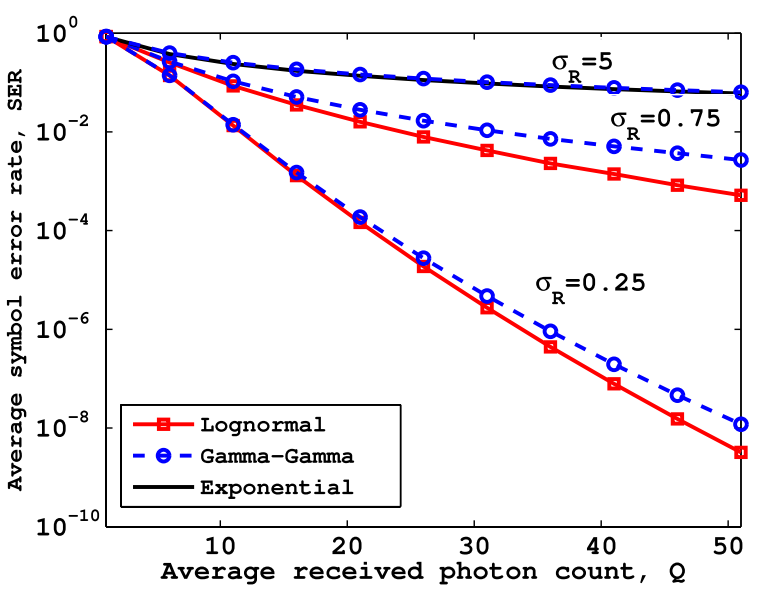

Fig. 2. Average SER versus average received signal photon count $Q$ using the approximate method for different channel models with $M=8, w=4$, and $K_{b}=1$.

turbulence, respectively. As expected, at strong turbulence with $\sigma_{R}=5$, the gamma-gamma model approaches the exponential model. On the other hand, the gamma-gamma model approaches the lognormal one as $\sigma_{R}$ decreases. So at weak turbulence $\left(\sigma_{R}=0.25\right)$, the gamma-gamma model reflects more the lognormal model than that at moderate turbulence $\left(\sigma_{R}=0.75\right)$.

\section{THERMAL-NOISE-LIMITED RECEIVERS}

In this section, we derive an expression for the upper bound of the average symbol-error rate of thermal-noise-limited MPPM systems in gamma-gamma channels. Thermal noise arises from photodetector's load resistor and amplifier noise. Under mild conditions, where there is no need for high sensitive receivers, thermal noise normally dominates shot noise. Indeed, in most practical systems, pin photodetectors are normally used rather than stringent quantum-limited photodectors. Thermal noise is well described by Gaussian statistics with zero mean and variance $\sigma_{n}^{2}$.

\section{A. System Model}

At the receiver side of the system, the photodiode converts the received optical intensity variations into corresponding variations in the electrical domain. The received electrical signal $y$ can be written as:

$$
y=\mathcal{R} \frac{M}{w} P_{R} C+n
$$

where $P_{R}$ is the average received signal power per symbol, $\mathcal{R}$ is the responsivity of the photodiode, $n$ is a signal-independent zero-mean white Gaussian noise with variance $\sigma_{n}^{2}$, and $C$ equals one for signal time slot and zero for non-signal time slot. The average power $P_{R}$ can be obtained by the link range equation [21]:

$$
P_{R}(h)=P_{T} \eta_{T} \eta_{R} G_{T} G_{R}\left(\frac{\lambda}{4 \pi L}\right)^{2} h
$$

where $P_{T}$ is the average transmitted power, $\eta_{T}$ and $\eta_{R}$ are the efficiencies of both the transmitter and receiver optics, 
respectively, $G_{T}$ and $G_{R}$ are both the transmitter and receiver telescope gains, respectively, $\lambda$ is the operating wavelength, $L$ is the distance between the transmitter and receiver, and $h$ is the channel state due to atmospheric turbulence, defined as $h \stackrel{\text { def }}{=} I / I_{0}$. From (7), the pdf of the channel state is thus

$$
\begin{aligned}
f(h) & =I_{0} g\left(I_{0} h\right) \\
& =\frac{2(\alpha \beta)^{\frac{\alpha+\beta}{2}}}{\Gamma(\alpha) \Gamma(\beta)} h^{\frac{\alpha+\beta}{2}-1} K_{\alpha-\beta}(2 \sqrt{\alpha \beta h}), \quad h \geq 0 .
\end{aligned}
$$

In our analysis below, we assume that $\eta_{T}=\eta_{R}=\eta$. In addition, we assume that the transmitter and receiver have the same telescopic gain, given by [21]:

$$
G_{T}=G_{R} \approx\left(\frac{\pi D}{\lambda}\right)^{2}
$$

where $D$ is the telescopic diameter. Substituting (26) into (24), we get

$$
P_{R}(h)=P_{T}\left(\frac{\eta A}{\lambda L}\right)^{2} h
$$

where $A=\pi D^{2} / 4$ is the transceiver telescopic area.

\section{B. Average Symbol Error Rate Upper Bound for MPPM}

As mentioned earlier, an MPPM symbol duration $T$ is divided into $M$ time slots, out of them only $w \in\{1,2, \ldots, M / 2\}$ time slots contain optical pulses. During a time frame, $T$, the MPPM signal current at the photodiode output can be written as

$$
x(t)=\frac{M \mathcal{R} P_{R}}{w} \sum_{k=0}^{M-1} C_{k} \operatorname{rect}\left(t-\frac{k T}{M}\right)
$$

where

$$
\operatorname{rect}(t)= \begin{cases}1, & \text { if } 0 \leq t<\frac{T}{M} \\ 0, & \text { otherwise }\end{cases}
$$

$C_{k}$ equals one for signal time slot and zero for nonsignal time slot. Using a union bound [22], we get an upper bound of the SER of MPPM as:

$$
P_{e \text { Sym }} \leq \frac{\left(\begin{array}{c}
M \\
w
\end{array}\right)-1}{2} \operatorname{erfc}\left(\frac{d_{\min } \sqrt{R_{b}}}{2 \sqrt{2} \sigma_{n}}\right)
$$

where $P_{e \mathrm{Sym}}$ is the SER, $R_{b}$ is the transmission bit rate, and $d_{\min }$ is the minimum Euclidean distance between two points in the signal constellation, defined as:

$$
d_{\text {min }}^{2} \stackrel{\text { def }}{=} \min _{\substack{x(t), z(t) \in \mathcal{S}(M, w): \\ x(t) \neq z(t)}} \int_{T}[x(t)-z(t)]^{2} d t
$$

where $\mathcal{S}(M, w)$ is the set of all possible MPPM signal currents. Using (28) and (30), we get $d_{\mathrm{min}}$ as follows:

$$
d_{\min }=\frac{\mathcal{R} P_{R}}{w} \sqrt{\frac{2 M \log _{2}\left(\begin{array}{c}
M \\
w
\end{array}\right)}{R_{b}}} .
$$

The effect of the atmospheric turbulence is taken into consideration by substituting for $P_{R}$ from (27). Thus, for a given channel state $h,(29)$ becomes:

$$
\begin{aligned}
P_{\text {Sym }}(h) \leq & \frac{\left(\begin{array}{c}
M \\
w
\end{array}\right)-1}{2} \\
& \times \operatorname{erfc}\left(\frac{\mathcal{R} P_{T}\left(\frac{\eta A}{\lambda L}\right)^{2} h}{2 w \sigma_{n}} \sqrt{M \log _{2}\left(\begin{array}{c}
M \\
w
\end{array}\right)}\right) .
\end{aligned}
$$

The average symbol-error rate $P_{e S y m}$ is obtained by averaging the last equation over $f(h)$ given in (25):

$$
P_{\text {Sym }}=\int_{0}^{\infty} P_{e \text { Sym }}(h) f(h) d h .
$$

Substituting (25) and (32) into (33) and expressing $K_{v}(\cdot)$ and $\operatorname{erfc}(\cdot)$ in terms of the Meijer G function [24], we get:

$$
\begin{aligned}
& P_{e \text { Sym }} \leq \frac{\left(\left(\begin{array}{c}
M \\
w
\end{array}\right)-1\right)(\alpha \beta)^{\frac{\alpha+\beta}{2}}}{2 \sqrt{\pi} \Gamma(\alpha) \Gamma(\beta)} \int_{0}^{\infty} h^{\frac{\alpha+\beta}{2}-1} \\
& \quad \times G_{0,2}^{2,0}\left(\alpha \beta h \mid \begin{array}{l}
\frac{\alpha-\beta}{2}, \frac{-\alpha+\beta}{2}
\end{array}\right) \\
& \quad \times G_{1,2}^{2,0}\left(\left\{\frac{\mathcal{R} P_{T}\left(\frac{\eta A}{\lambda L}\right)^{2} h}{2 w \sigma_{n}} \sqrt{M \log _{2}\left(\begin{array}{c}
M \\
w
\end{array}\right)}\right\}^{2} \mid \begin{array}{c}
1,- \\
0,0.5
\end{array}\right) d h .
\end{aligned}
$$

Finally, using the integral form for the Meijer G function [24], we get an upper bound (on the average SER for MPPM system) given in (35) at the bottom of this page.

\section{Numerical Results}

In this section, we use the upper bound in (35) to study the performance of thermal-noise-limited MPPM systems under gamma-gamma channels. Since the SER is dependent on the operating wavelength, it would be meaningful to use $C_{n}^{2}$ as a measure to the turbulence strength. In all our evaluations below, we use the same system parameters as in [23], listed in Table I again.

Fig. 3 shows the average SER versus average transmitted power of the MPPM system at different atmospheric turbulence strengths $\left(C_{n}^{2}=5.2 \times 10^{-16} \mathrm{~m}^{-2 / 3}, C_{n}^{2}=2.1 \times\right.$

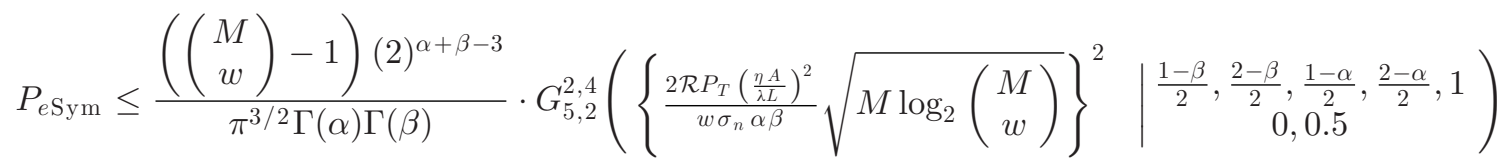


TABLE I

SYSTEM PARAMETERS

\begin{tabular}{|c|c|c|}
\hline Parameter & Symbol & Value \\
\hline Tx/Rx Optics efficiency & $\eta$ & 0.8 \\
\hline Photodiode resposivity & $\mathcal{R}$ & $0.5 \mathrm{~A} / \mathrm{W}$ \\
\hline Transmitter and receiver telescopic diameter & $D$ & $8 \mathrm{~cm}$ \\
\hline Distance & $L$ & $5 \mathrm{~km}$ \\
\hline
\end{tabular}

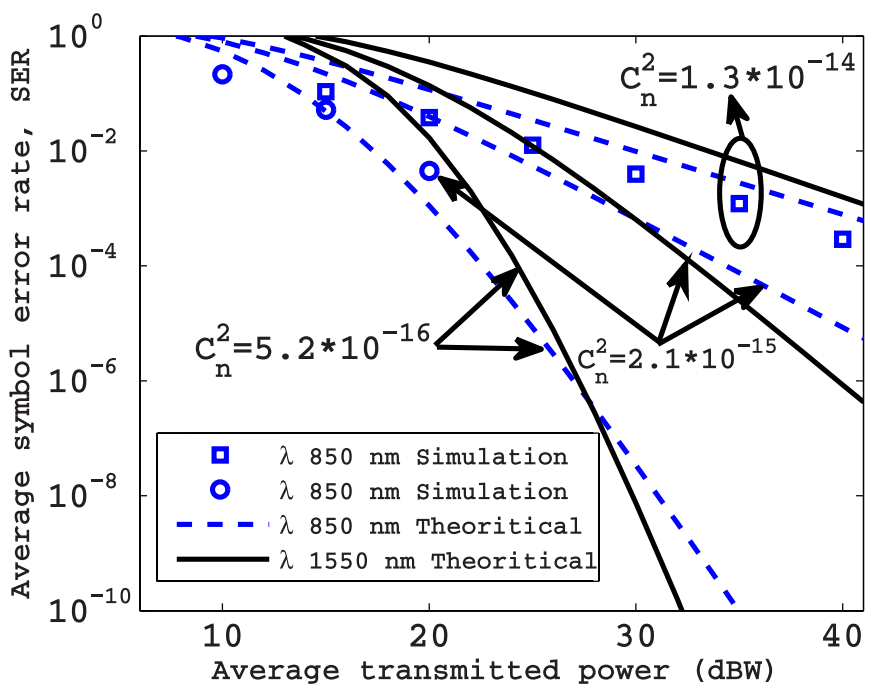

Fig. 3. Average SER versus average transmitted power in dBW for MPPM system with $M=4, w=2$ and $\sigma_{n}^{2}=3.162 A^{2}$ under different turbulence strengths for both $\lambda=850 \mathrm{~nm}$ and $\lambda=1550 \mathrm{~nm}$.

$10^{-15} \mathrm{~m}^{-2 / 3}$, and $C_{n}^{2}=1.3 \times 10^{-14} \mathrm{~m}^{-2 / 3}$, for weak, moderate, and strong turbulence, respectively) and two operating wavelengths $(\lambda=850 \mathrm{~nm}$ and $\lambda=1550 \mathrm{~nm})$. The theoretical results for strong and moderate turbulence are validated by Monte Carlo simulation results. However, we did not validate theoretical results at weak turbulence due to the very long simulation running time at weak turbulence. It should be noticed that for nonturbulent channels, the performance of systems with short wavelengths is better than that of systems with long wavelengths over all values of the average transmitted power. The reason for that is the inverse proportionality between the telescopic gain and the square of the operational wavelength as shown in (26). However, as shown in Fig. 3, in the case of turbulence, the performance of systems with short wavelengths is better than that of systems with long wavelengths at low power only. This is because in the case of low power, the noise has a dominant effect and since short wavelengths introduce higher gain than long wavelengths, the performance of short wavelength systems will be better. On the other hand, as the power increases above a certain threshold, the turbulence effect dominates and since $\sigma_{R}^{2}$ is inversely proportional to $\lambda^{7 / 6}$ (for fixed $C_{n}^{2}$ ) the turbulence would have more effect on short-wavelength systems, and hence, the performance of long-wavelength systems will be better. Specifically for the values of $C_{n}^{2}$ in Fig. 3, the scintillation index at short wavelengths is $0.359,0.9$, and 1.24 (for weak, moderate, and strong turbulence, respectively), while at long wavelengths this index reduces to $0.19,0.61$, and 1.2 (for weak, moderate, and strong turbulence, respectively).

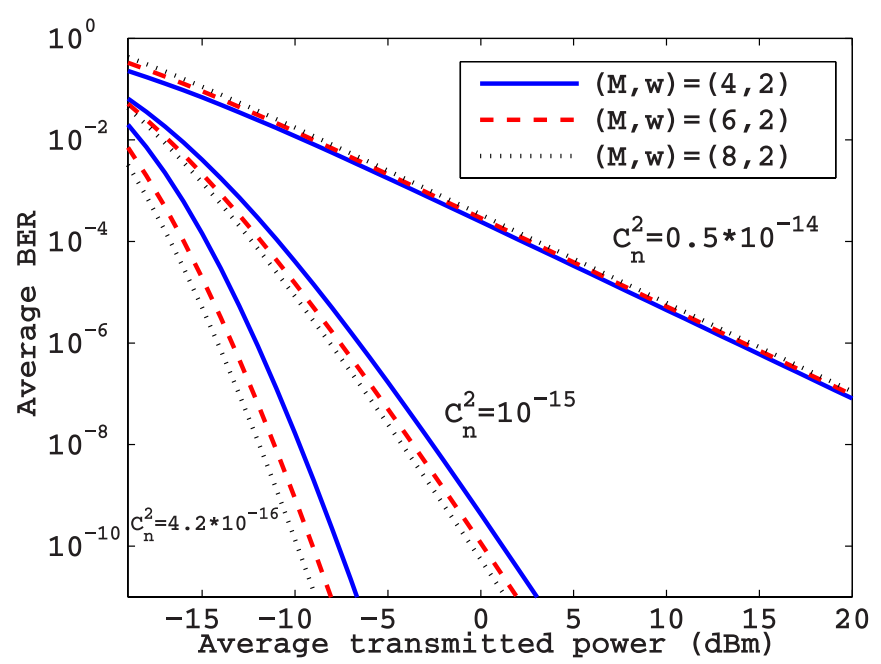

Fig. 4. Average BER versus average transmitted power per bit in $\mathrm{dBm}$ for MPPM systems with $(M, w)=\{(4,2),(6,2),(8,2)\}$ and $\sigma_{n}^{2}=25 \times$ $10^{-14} A^{2}$ at different atmospheric turbulence strengths for $\lambda=1550 \mathrm{~nm}$.

Notice also that for strong turbulence, both scintillation indices are close to each other (1.24 and 1.2), which explains the small difference in the corresponding curves of the figure.

Fig. 4 shows the effect of increasing the number of time slots per symbol (modulation level) on the performance of MPPM-FSO systems under the constraints of same transmission rate, average power per bit, and bandwidth. It can be seen from the figure that increasing the number of time slots per symbol (under the above constraints) would improve the system performance for both weak and moderate turbulence only, while the system performance cannot be improved in strong turbulence. This is because for both weak and moderate turbulence, the noise has the dominant effect and as the modulation level increases, the peak power increases as well leading to higher signal-to-noise ratios and improved BERs. For the case of strong turbulence, however, the turbulence has the dominant effect and so the performance cannot be improved by increasing the modulation level.

\section{COMPARISON BETWEen THE BER FOR BOTH MPPM-AND PPM-FSO SYSTEMS}

In this section, we make comparisons between the performance of FSO systems adopting MPPM techniques and that adopting PPM techniques under different atmospheric turbulence conditions for both shot- and thermal-noise limited systems. The number of time slots for both PPM and MPPM systems have been chosen so as they transmit same data rates for fixed bandwidth. Also the comparison has been made with the same average energy per bit. It can be seen from Figs. 5 and 6 that under the aforementioned conditions MPPM systems perform better than PPM systems for weak turbulence. However, at moderate and strong turbulence, both systems perform almost similarly in the shot-noise limited case, while PPM system performs better in the thermal-noise limited case. Indeed, in the case of transmitting the same data rate with the same average energy per bit, MPPM systems have higher peak power per slot 


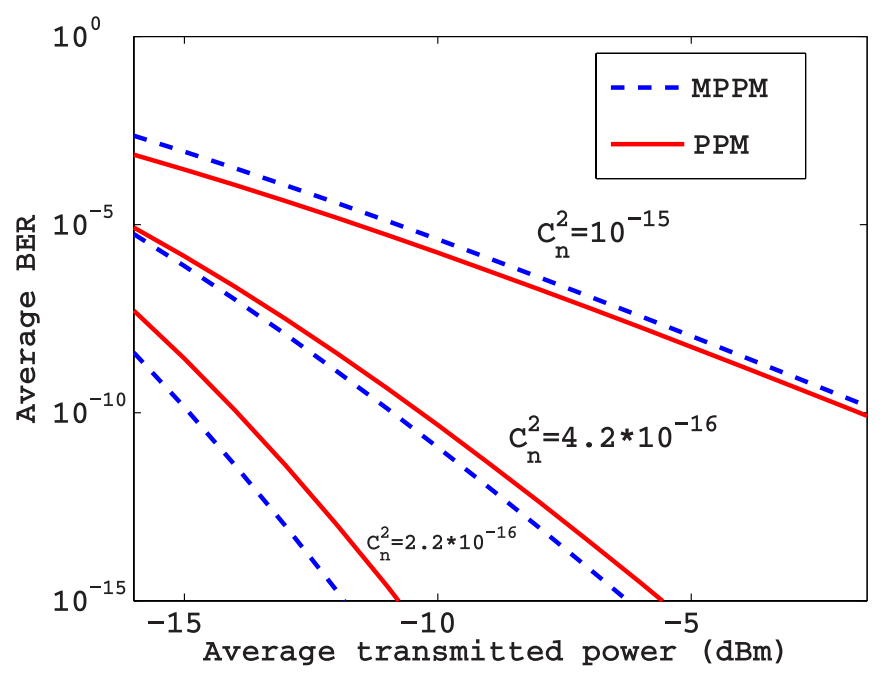

Fig. 5. Comparison between the performance of MPPM techniques with $(M, w)=(12,2)$ and PPM techniques with four time slots in the case of thermal-noise limited systems with $\sigma_{n}^{2}=25 \times 10^{-14} A^{2}$ under different turbulence strengths for $\lambda=1550 \mathrm{~nm}$ and equal transmission rates.

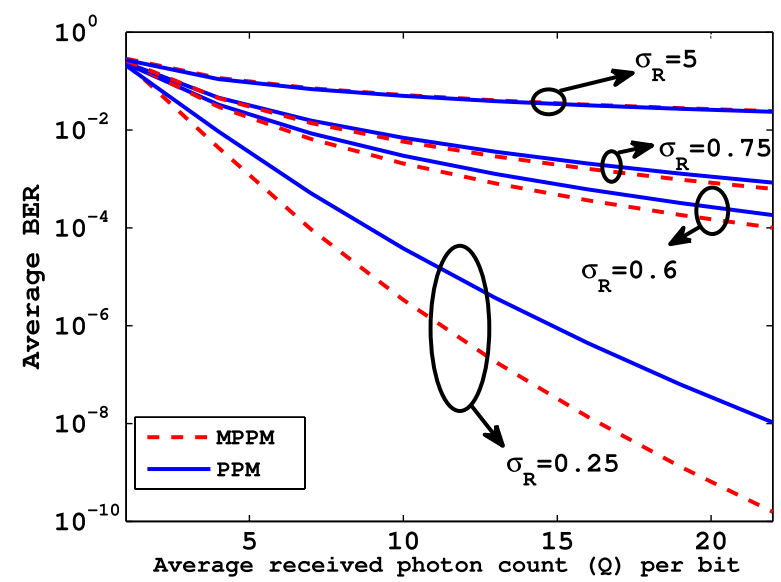

Fig. 6. Comparison between the performance of MPPM techniques with $(M, w)=(12,2)$ and PPM techniques with four time slots in the case of shot-noise limited systems under different turbulence strengths for equal transmission rates.

as compared to PPM systems. Again, this leads to higher signalto-noise ratios and improved BERs of the corresponding FSO systems. On the other hand, at strong turbulence, the turbulence effect dominates.

\section{CONCLUSION}

The performance of FSO communication systems adopting MPPM techniques has been investigated taking into account the effects of both the atmospheric turbulence and receiver noise. Gamma-gamma channels have been assumed as atmospheric turbulence models. Both shot- and thermal-noise limited scenarios have been considered at the receiver side. Both exact and approximate expressions of the average symbol-error rate (SER) have been derived for the shot-noise limited system, while a closed form for the upper bound of the average SER, based on the Meijer $\mathrm{G}$ function, has been derived for the thermal-noise limited system. The SER performance of the above shot-noise limited system has been compared to corresponding systems working in both lognormal and exponential channels. The effects of changing the atmospheric conditions, operational wavelengths, and number of time slots on the average system performance have been studied for the thermal-noise limited system. Our results reveal that the SER performance of short-wavelength systems is better than that of long-wavelength systems at low power, whereas as the power increases above a certain threshold, the above performance conclusion reverses. Furthermore, for both low and moderate turbulence, increasing the modulation level improves the system performance, while for strong turbulence, the system performance cannot be improved by increasing modulation level. Finally, the BER performance of the MPPM system, in a gamma-gamma channel, has been compared with that of the traditional PPM technique. Both shot- and thermal-noise limited systems have been studied under same constraints on the average energy per bit, transmission data rate, and bandwidth. Our results reveal that at weak turbulence, the performances of the MPPM systems are better than that of corresponding PPM systems. However, at moderate and strong turbulence, both systems perform almost similarly in the shotnoise limited case, while PPM system performs better in the thermal-noise limited case.

\section{ACKNOWLEDGMENT}

The authors would like to thank Egyptian Ministry of Higher Education (MoHE) for its support.

\section{REFERENCES}

[1] S. S. Muhammad, T. Javornik, I. Jelovcan, E. Leitgeb, and O. Koudelka "Reed-Solomon coded PPM for terrestrial FSO links," in Proc. Int. Conf. Elect. Eng., Apr. 2007, pp. 1-5.

[2] X. Zhu and J. M. Kahn, "Free-space optical communication through atmospheric turbulence channels," IEEE Trans. Commun., vol. 50, no. 8, pp. 1293-1300, Aug. 2002.

[3] Z. Ghassemlooy, W. Popoola, and S. Rajbhandari, Optical Wireless Communications: System and Channel Modelling With MATLAB, 1st ed. Boca Raton, FL, USA: CRC Press, Aug. 2012.

[4] T. Nguyen and L. Lampe, "Coded multipulse pulse-position modulation for free-space optical communications," IEEE Trans. Commun., vol. 58, no. 4, pp. 1036-1041, Apr. 2010.

[5] S. J. Dolinar, J. Hamkins, B. E. Moision, and V. A. Vilnrotter, "Optical modulation and coding," in Deep Space Optical Communications, 1st ed., H. Hemmati, Ed. New York, NY, USA: Wiley-Interscience, Apr. 2006.

[6] C. N. Georghiades, "Modulation and coding for throughput -efficient optical systems," IEEE Trans. Inf. Theory, vol. 40, no. 5, pp. 1313-1326, Sep. 1994.

[7] H. Sugiyama and K. Nosu, "MPPM: A method for improving the band utilization efficiency in optical PPM," J. Lightw. Technol., vol. 7, no. 3, pp. 465-472, Mar. 1989.

[8] N. Aoki, T. Ohtsuki, and I. Sasase, "Performance analysis of multi-pulse pulse position modulation using avalanche photodiode in optical intersatellite links," IEICE Trans. Commun., vol. E79-B, no. 1, pp. 52-56, Jan. 1996.

[9] J. Hamkins and B. Moision, "Multipulse pulse-position modulation on discrete memoryless channels," Interplanetary Netw. Progr. Rep., vol. 42, no. 161, pp. 1-13, May 2005.

[10] W. Gappmair and S. S. Muhammad, "Error performance of PPM/Poisson channels in turbulent atmosphere with gamma-gamma distribution," Electron. Lett., vol. 43, no. 16, pp. 2-3, Aug. 2007.

[11] F. Xu, M. A. Khalighi, and S. Bourennane, "Coded PPM and multipulse PPM and iterative detection for free-space optical links," J. Opt. Commun. Netw., vol. 1, no. 5, pp. 404-415, Oct. 2009. 
[12] F. Xu, M. Khalighi, and S. Bourennane, "Efficient channel coding for multipulse pulse position modulation in terrestrial FSO systems," Proc. SPIE, vol. 7464, pp. 74 640M-1-74 640M-12, Aug. 2009.

[13] S. G. Wilson, M. B. Pearce, Q. Cao, and M. Baedke, "Optical repetition MIMO transmission with multipulse PPM," IEEE J. Sel. Areas Commun., vol. 23, no. 9, pp. 1901-1910, Sep. 2005.

[14] J. M. G. Balsells, A. J. Navas, J. F. Paris, M. C. Vazquez, and A. P. Notario, "Closed-form BER analysis of variable weight MPPM coding under gamma-gamma scintillation for atmospheric optical communications," Opt. Lett., vol. 37, no. 4, pp. 719-721, Feb. 2012.

[15] R. L. Al-Habash, M. A. Andrews, and L. C. Phillips, "Mathematical model for the irradiance probability density function of a laser beam propagating through turbulent media," Opt. Eng., vol. 40, no. 8, pp. 1554 1562, Aug. 2001.

[16] K. Kiasaleh, "Performance of APD-based, PPM free-space optical communication systems in atmospheric turbulence," IEEE Trans. Commun., vol. 53 , no. 9, pp. 1455-1461, Sep. 2005.

[17] K. Kikuchi, T. Okoshi, and A. Hirose, "Achievement of shot-noise-limited sensitivity and 50-db dynamic range by photon-counting receiver using SI avalanche photodiode," J. Lightw. Technol., vol. LT-4, no. 7, pp. 828-832, Jul. 1986.

[18] S. Dolinar, B. I. Erkmen, B. Moision, K. M. Birnbaum, and D. Divsalar, "The ultimate limits of optical communication efficiency with photon-counting receivers," in Proc. IEEE Int. Symp. Inf. Theory, 2012, pp. 541-545.

[19] I. S. Gradshteyn and I. M. Ryzhik, in Table of Integrals, Series, and Products, A. Jeffrey and D. Zwillinger, Eds., 7th ed. ed. New York, NY, USA: Academic, Mar. 2007.

[20] M. Abramowitz and I. A. Stegun, Eds., Handbook of Mathematical Functions, 10th ed. ed.. $\quad$ New York, NY, USA: Dover, Dec. 1972.

[21] A. Polishuk and S. Arnon, "Optimization of a laser satellite communication system with an optical preamplifier," J. Opt. Soc. Amer. A, Opt., Image Sci., Vis., vol. 21, no. 7, pp. 1307-1315, Jul. 2004.

[22] S. Haykin, Communication Systems, 4th ed. ed. New York, NY, USA Wiley, 2001

[23] X. Yi, Z. Liu, P. Yue, and T. Shang, "BER performance analysis for M-ary PPM over gamma-gamma atmospheric turbulence channels," in Proc. 6th Wireless Commun. Network. Mobile Comput., Sep. 2010, pp. 1-4.

[24] Wolfram Function Site. (2013, Feb.). [Online]. Available:http://functions. wolfram.com/

Ahmed E. Morra was born in Menufiya, Egypt, in 1984. He received the B.S. and M.S. degrees from the Faculty of Electronic Engineering, Menufiya University, Menouf 32952, Egypt, in 2006 and 2010, respectively. In 2006, he joined the Electronics and Electrical Communications Engineering Department, Menufiya University, Menufia, Egypt, and was promoted to the position of Lecturer Assistant in 2011. He is currently on leave from the Menufiya University and is working toward the Ph.D. degree with the Department of Electronics and Communications Engineering, School of Electronics, Communications, and Computer Engineering, Egypt-Japan University of Science and Technology, Alexandria, Egypt. His research interests include optical communications, free-space optical communications systems, and optoelectronics.

Haitham S. Khallaf was born in Tanta, Egypt, in 1980. He received the B.S degree from the Faculty of Electronic Engineering, Menufiya University, Menufia, Egypt, in 2005 and the M.S. degree from Egypt-Japan University of Science and Technology (E-JUST), Alexandria, Egypt, in 2013. In 2006, he joined the Electrical and Communications Engineering Department, Menufiya University. After that, he joined Nuclear Research Center (NRC),Egypt. He is currently on leave from NRC and is working toward the Ph.D. degree with the Department of Electronics and Communications Engineering, School of Electronics, Communications, and Computer Engineering, E-JUST. His research interests include optical communications and integration between optical and wireless systems.
Hossam M. H. Shalaby (S'83-M'91-SM'99) was born in Giza, Egypt, in 1961. He received the B.S. and M.S. degrees from Alexandria University, Alexandria, Egypt, in 1983 and 1986, respectively, and the Ph.D. degree from the University of Maryland, College Park, MD, USA, in 1991, all in electrical engineering. In 1991, he joined the Electrical Engineering Department, Alexandria University, and was promoted to a Professor in 2001. He is currently on leave from Alexandria University and is the Chair of the Department of Electronics and Communications Engineering, School of Electronics, Communications, and Computer Engineering, Egypt-Japan University of Science and Technology, Alexandria, Egypt. From December 2000 to 2004, he was an Adjunct Professor with the Faculty of Sciences and Engineering, Department of Electrical and Information Engineering, Laval University, Quebec, QC, Canada. From September 1996 to February 2001, he was on leave from the Alexandria University. From September 1996 to January 1998, he was with the Electrical and Computer Engineering Department, International Islamic University Malaysia, and from February 1998 to February 2001, he was with the School of Electrical and Electronic Engineering, Nanyang Technological University, Singapore. His research interests include optical communications, optical CDMA, optical burst-switching, OFDM technology, and information theory. He has served as a Student Branch Counselor at Alexandria University, the IEEE Alexandria and North Delta Subsection, from 2002 to 2006, and served as a Chairman of the student activities committee of the IEEE Alexandria Subsection from 1995 to 1996. He received an SRC Fellowship from 1987 to 1991 from Systems Research Center, Maryland; the State Excellence Award in Engineering Sciences in 2007 from the Academy of Scientific Research and Technology, Egypt; the Shoman Prize for Young Arab Researchers in 2002 from Abdul HameedShoman Foundation, Amman, Jordan; the State Incentive Award in Engineering Sciences in 1995 and 2001 from Academy of Scientific Research and Technology, Egypt; the University Excellence Award in 2009 from Alexandria University; and the University Incentive Award in 1996 from Alexandria University. He is a Senior Member of both the IEEE Photonics Society and The Optical Society of America (OSA)

Zen Kawasaki (M'72-SM'01) received the Bachelor, Master, and Ph.D degrees from the Graduate School of Engineering, Osaka University, Suita, Japan, in 1973, 1975, and 1978, respectively. He started his career as Research Associate at Nagoya University, Nagoya, Japan, from 1979. He moved to Osaka University in 1989 to be a Lecturer, where he was promoted as the Associate Professor and Full Professor of Department of Electrical, Electronics and Information Engineering, Graduate School of Engineering in 1991 and 2000, respectively. $\mathrm{He}$ is the Fellow of the Institute of Electrical Engineering Japan. He is currently the President of the International Committee of Atmospheric Electricity. 